\title{
Process water conditioning to improve slurry dewatering
}

\author{
AJ Vietti Vietti Slurrytec (Pty) Ltd, South Africa \\ SL Rabie Vietti Slurrytec (Pty) Ltd, South Africa \\ K Ntshabele Debswana Diamond Company (Pty) Ltd, Botswana
}

Portions of this paper were originally published in 'A strategy for improving water recovery in kimberlitic diamond mines' as it appeared in the February 2019 Edition of the SAIMM Journal, Volume 119 published by The Southern African Institute of Mining and Metallurgy.

\begin{abstract}
Mine site location and the sources from which raw water is drawn are fixed by geology, geography and climate. The colloidal behaviour of the tailings is therefore randomly determined by the tailings mineralogy and the chemical characteristics of the process water circuit.

In some fortunate cases, the tailings slurries are non-dispersive and solid/liquid separation, either by gravity thickening or filtration, is easily achieved at low capital and operating cost. In less fortunate cases the tailings are highly dispersive and solid/liquid separation is achieved only at high capital and operating cost.

This paper presents a strategy by which difficult-to-treat slurries can be modified by conditioning the process water circuit with a process water conditioner (ClariVie44 ${ }^{\circledR}$ ) so that gravity thickening and filtration can be enhanced.
\end{abstract}

Keywords: clays, colloidal, filtration, thickening

\section{Introduction}

Each mineral deposit has a unique mineralogy and chemistry and likewise, the raw water source supplying the potential mine will have a unique chemical signature. Consequently, the colloidal properties of slurries generated from these two components may, by chance, be favourable for settling and solid/liquid separation or they may not.

Water is used in the metallurgical process to assist in washing away gangue minerals and concentrating the valuable products. Most mineral deposits contain a degree of clay minerals which, when wetted with the plant process water, can generate potentially settling or non-settling slurries determined largely by the interaction between clay mineralogy and process water chemical constituents.

In cases where the colloidal properties of a tailings slurry favours settling, dewatering processes are relatively efficient and simple, practical steps, such as improving the flocculant reagent type, make-up and dosing and focusing on unit process control, are the only requirements for enhancing water recovery or underflow density.

At other mine sites where the colloidal properties of the tailings encourage dispersion, solid/liquid separation at the dewatering processes may not be achieved at all, in which case efforts for improving water recovery by increasing reagent type or dose would have limited effect. Consequently, controlling the process water circuit chemical condition is the first step in improving plant water recovery in these cases.

A laboratory trial was conducted to determine the improvement in efficiency of two dewatering unit processes (namely gravity paste thickening and pressure filtration) when treating tailings slurries which had been generated under controlled and uncontrolled dispersive states by treating the plant circuit water with a process water conditioner. 


\section{Controlled and uncontrolled slurry dispersions}

Within a metallurgical circuit, the first contact between newly mined ore and the plant process water is typically at the milling or scrubbing unit process. This step is critical in determining the colloidal state of the resulting slurry since the clays within the ore absorb water and other polar molecules between their unit layers which initiates swelling. Once the clays have undergone swelling and delamination, the process is irreversible and therefore it is this first contact that needs to be controlled. The degree of swelling depends largely on the nature and concentration of the cations contained in the contacting water and the clay types involved (Sequet et al. 1975; Mering 1946).

Monovalent cations in low concentration tend to cause unlimited swelling; a condition commonly referred to as uncontrolled dispersion. Monovalent cations having small hydration shells can penetrate easily into the interlayer spaces between adjacent clay platelets, which are typically occupied by two or three stacked layers of water molecules which themselves are bound to the clay platelet surfaces. Once in the interlayer position, the cations can draw in more water molecules by osmotic action and therefore, initiate hydraulic swelling. Divalent cations on the other hand tend to cause limited swelling since they have a disruptive effect on the interlayer water layer structure and they can provide links between charged sites on adjacent silicate sheets. This condition is commonly referred to as controlled dispersion (Figure 1).

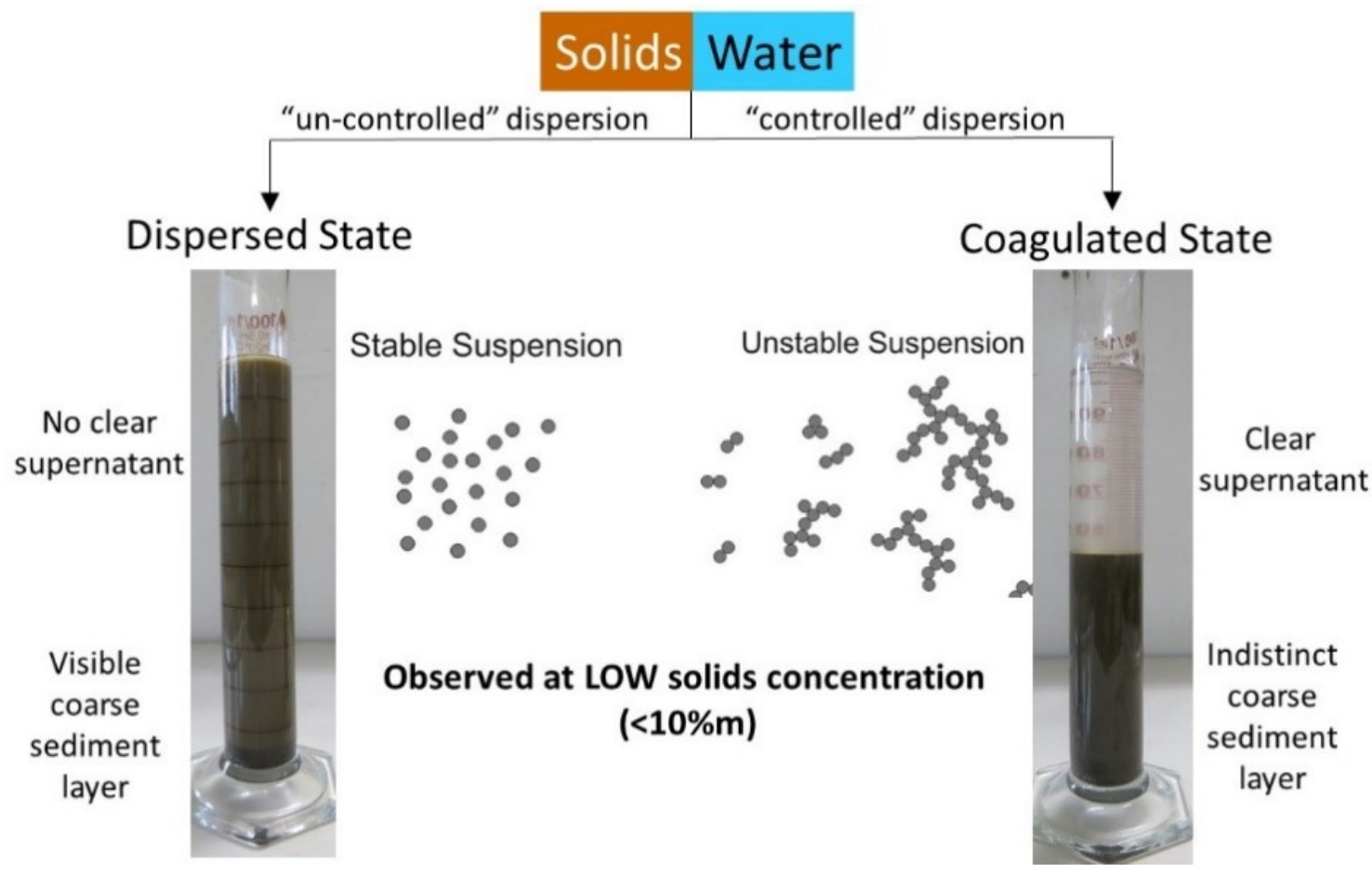

Figure 1 Dispersive slurry (left) and coagulated slurry (right) after uncontrolled and controlled dispersion (Vietti 2018)

From a practical point of view, mines which draw their raw water from surface sources such as rivers, dams or lakes will tend to introduce low salinity water into the circuit, which in turn will result in the process water circuit having a relatively low cation content and low conductivity. This quality of water will promote uncontrolled dispersive conditions and the resulting slurry will tend to be poorly or non-receptive to polymer flocculant dosing and even after excessively high doses, the thickener overflow clarity is generally poor. It is usually necessary to dose a polymer coagulant before flocculant addition to achieve good solid/liquid separation with good overflow clarity. The unintended consequences are the excess entrained polymer and higher operating costs.

On the other hand, mines which draw their raw water from deep well-field, brackish or sea water sources will have process water circuits which have elevated cation contents with high conductivities. This quality of 
process water inhibits clay swelling and dispersion, resulting in slurries which are easily flocculated at low doses without the need for additional polymer coagulant reagent. Thickener overflows will have excellent clarity while underflow densities will be maximised at minimal flocculant dose.

As the salinity of a slurry increases, a critical coagulation concentration (CCC) is reached which differentiates between dispersive and coagulated colloidal states (Figure 2 ). The slurry salinity can be determined by simply measuring its conductivity (in $\mathrm{mS} / \mathrm{cm}$ ) and it is a measurable parameter that can be used in a feed-back loop to maintain the CCC at which a process water circuit should be maintained to ensure that controlled dispersion always occurs. The CCC forms the basis of the process water conditioning strategy for improving mine water recovery.
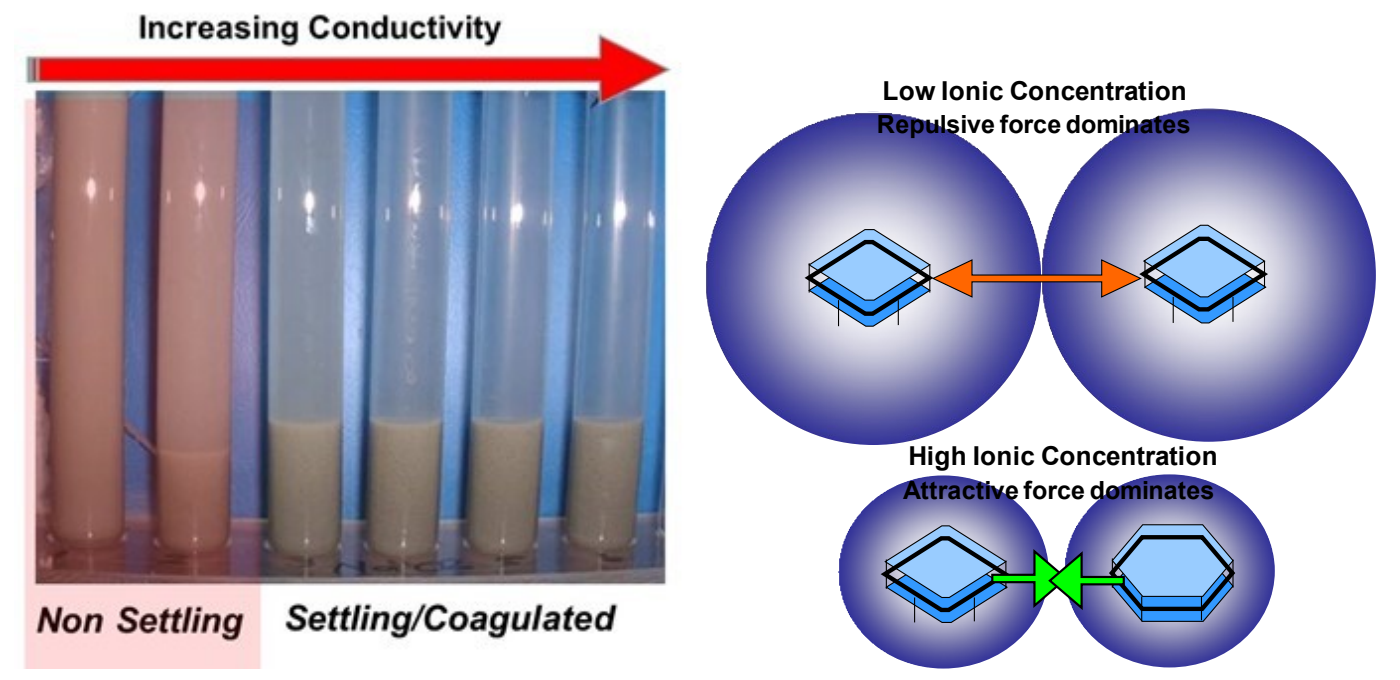

Figure 2 Effect of increasing salinity on the suspension behaviour of a tailing's slurry (Vietti 2018)

\section{Process water conditioning}

Vietti Slurrytec (VST) has developed the ClariVie $44^{\circledR}$ process water conditioner which is a blend of specific cations designed to increase the conductivity of a process water circuit without introducing environmental and corrosive pollutants (Vietti 2018). Since the process water conditioner is not polymer based, it is recycled in the process water circuit without the need for continuous dosing. ClariVie $44^{\circledR}$ is dosed into the process water circuit to increase the conductivity of the circuit and thereafter, smaller top-up doses are required to compensate for the diluting effect of the raw water make-up (Figure 3 ).

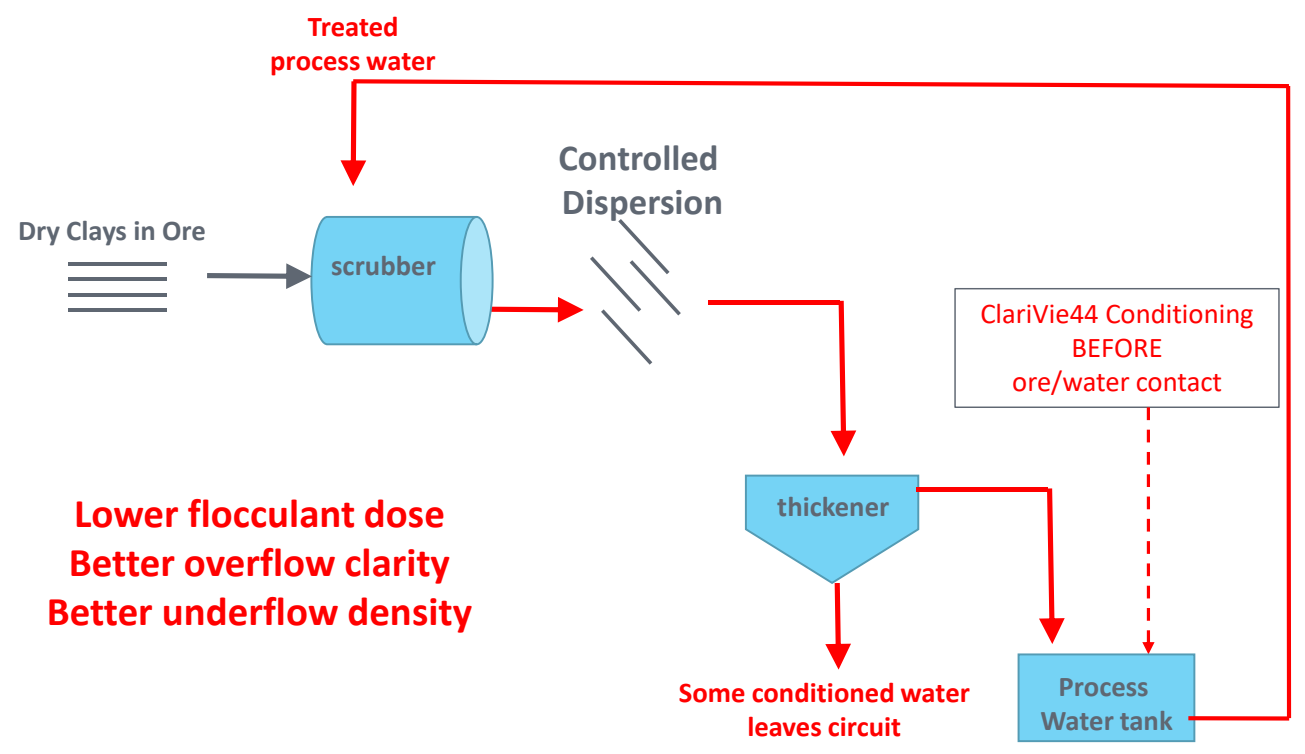

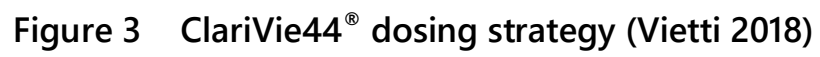


The strategy has been employed at four Southern African mines since January 2017 where raw water is sourced from low conductivity water sources. ClariVie $44{ }^{\circledR}$ has been implemented to condition the process water circuit to a minimum conductivity target, with excellent results showing a clear correlation between increasing process water conductivity and decreasing flocculant consumption (Figure 4).

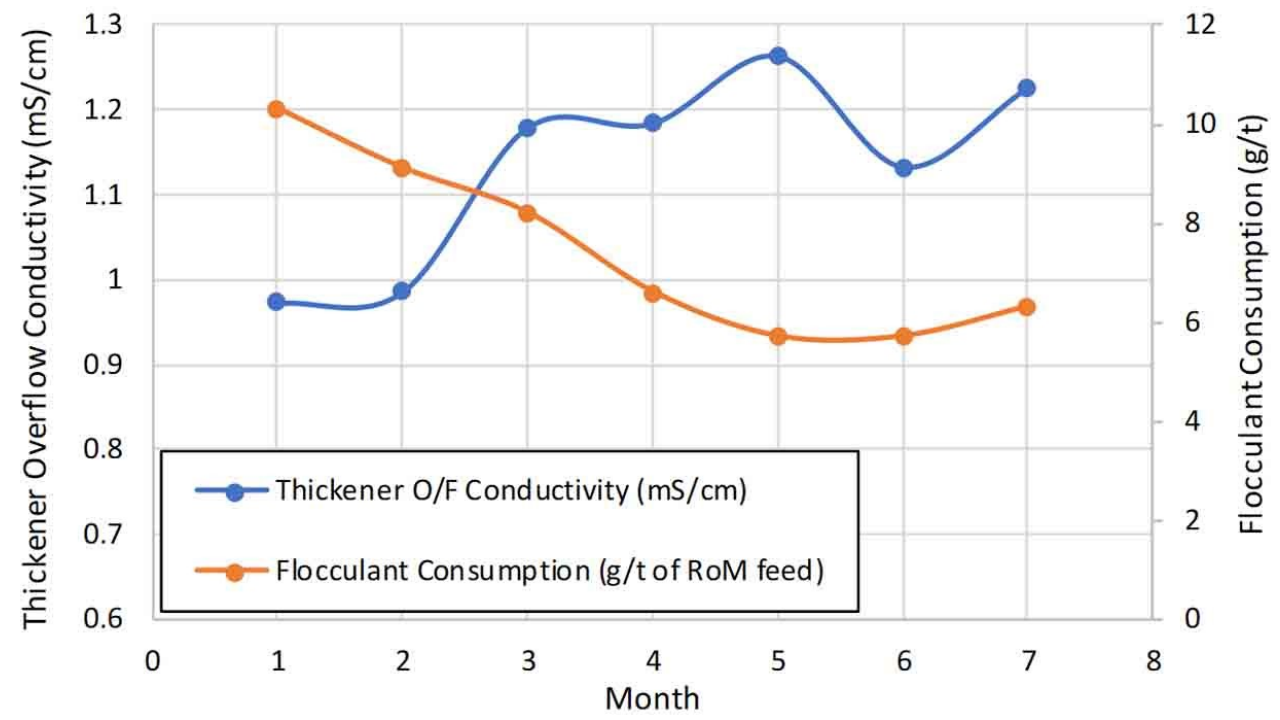

Figure 4 Correlation between process water conductivity and decreasing flocculant consumption at one site

To quantify the effectiveness of the process water conditioning strategy for improving dewatering, a laboratory trial was conducted on an ore source known to be difficult to treat derived from a kimberlite dump retreatment plant in Botswana.

\section{$4 \quad$ Laboratory dewatering trial}

A kimberlitic diamond mine in Botswana operates a separate re-treatment plant for the recovery of diamonds from dump material. The mine derives its raw water supply from shallow boreholes and consequently, the plant process water circuit has a low average conductivity. A single $25 \mathrm{~m}$ diameter paste thickener is used to dewater the tailings. Currently a relatively low underflow solids concentration ( 39 to $44 \% \mathrm{~m}$ ) is achieved, while overflow water clarity is generally poor and high flocculant doses are required to achieve relatively clear overflow quality.

For the test work, two feed slurries in different colloidal states were generated from dry crushed and screened $(-300 \mu \mathrm{m})$ dump ore material and the plant process water:

1. Unconditioned slurry - made up in low conductivity process water.

2. Conditioned slurry - made up in ClariVie $44^{\circledR}$ conditioned process water (dosed at $1.5 \mathrm{~kg} / \mathrm{m}^{3}$ ).

Both slurry types were then dewatered via two dewatering technologies in sequence at the laboratory scale, i.e. paste thickening of the $-300 \mu \mathrm{m}$ feed slurry, followed by pressure filtration of the thickened underflow combined with a coarse grits fraction $(+300-1,000 \mu \mathrm{m})$. Process-specific operational parameters were then compared to determine if conditioning of the process water had any effect on improving the dewatering performances of these unit processes. 


\subsection{Results}

\subsubsection{Slurry feed properties}

The properties of both thickener feed slurries are presented in Table 1.

Table 1 Thickener feed slurry properties

\begin{tabular}{lll}
\hline Parameter & Unconditioned & Conditioned \\
\hline $\mathrm{pH}$ & 8.3 & 7.9 \\
Conductivity $(\mathrm{mS} / \mathrm{cm})$ & 1.6 & 2.3 \\
Methylene blue index $(\mathrm{meq} / 100 \mathrm{~g})$ & 12.0 & 12.0 \\
Solids specific gravity $\left(\mathrm{g} / \mathrm{cm}^{3}\right)$ & 2.447 & 2.447 \\
Particle top size $(\mu \mathrm{m})$ & 300 & 300 \\
d$_{50}$ particle Size $(\mu \mathrm{m})$ & 37.2 & 37.2 \\
Smectite in clay fraction $(\% \mathrm{~m})$ & 38.1 & 38.1 \\
Talc in clay fraction $(\% \mathrm{~m})$ & 3.1 & 3.1 \\
\hline
\end{tabular}

An initial flocculant screening analysis using static cylinder tests was conducted on the feed slurries and M5250 supplied by BASF was found to be the best performing reagent on both slurry types (Table 2).

Table 2 M5250 flocculant dose results under static cylinder test conditions

\begin{tabular}{lllll}
\hline Slurry state & $\begin{array}{l}\text { Solids concentration } \\
(\% \mathrm{~m})\end{array}$ & $\begin{array}{l}\text { Flocculant } \\
\text { dose }(\mathbf{g} / \mathbf{t})\end{array}$ & $\begin{array}{l}\text { Settling rate } \\
(\mathbf{m} / \mathbf{h r})\end{array}$ & $\begin{array}{l}\text { Clarity } \\
\text { (wedge no.) }\end{array}$ \\
\hline Unconditioned & 7.5 & 90 & 35 & 36 \\
Conditioned & 7.5 & 50 & 20 & 48 \\
\hline
\end{tabular}

Note: Clarity wedge rating from 0 (poor) to 50 (clear).

\subsubsection{Paste thickening performance}

Dynamic thickening tests were conducted on both slurry feed samples at semi-pilot scale in a $190 \mathrm{~mm}$ diameter paste thickener (Figure 5) under the test conditions described in Table 3.

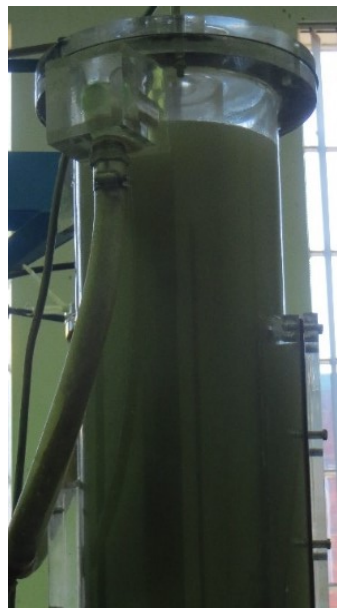

(a)

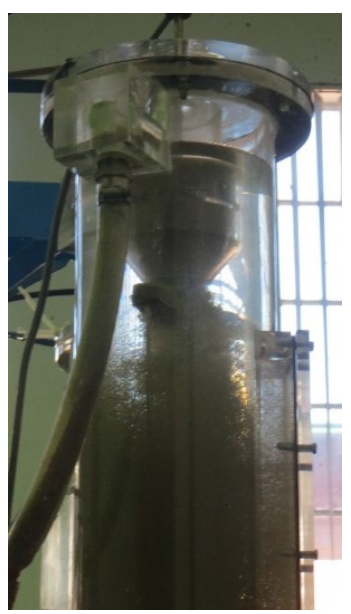

(b)

Figure 5 (a) Overflow clarity of unconditioned feed; and (b) Conditioned feed 
Table 3 Semi-pilot paste thickening test conditions

\begin{tabular}{lll}
\hline Parameter & Unconditioned & Conditioned \\
\hline Feed solids concentration $(\% \mathrm{~m})$ & 8.0 & 8.3 \\
Flocculant dose $(\mathrm{g} / \mathrm{t})$ & 140 & 80 \\
Final mud bed height $(\mathrm{mm})$ & 800 & 800 \\
Rake speed $(\mathrm{rpm})$ & 2 & 2 \\
Flux rate $\left(\mathrm{t} / \mathrm{m}^{2} . \mathrm{hr}\right)$ & 0.20 & 0.25 \\
\hline
\end{tabular}

The flocculant dose requirement was significantly lower (43\% less) and the overflow clarity was significantly better for the conditioned slurry than the unconditioned slurry, even though it was fed at a higher throughput to the thickener (Figures 6 and 7).

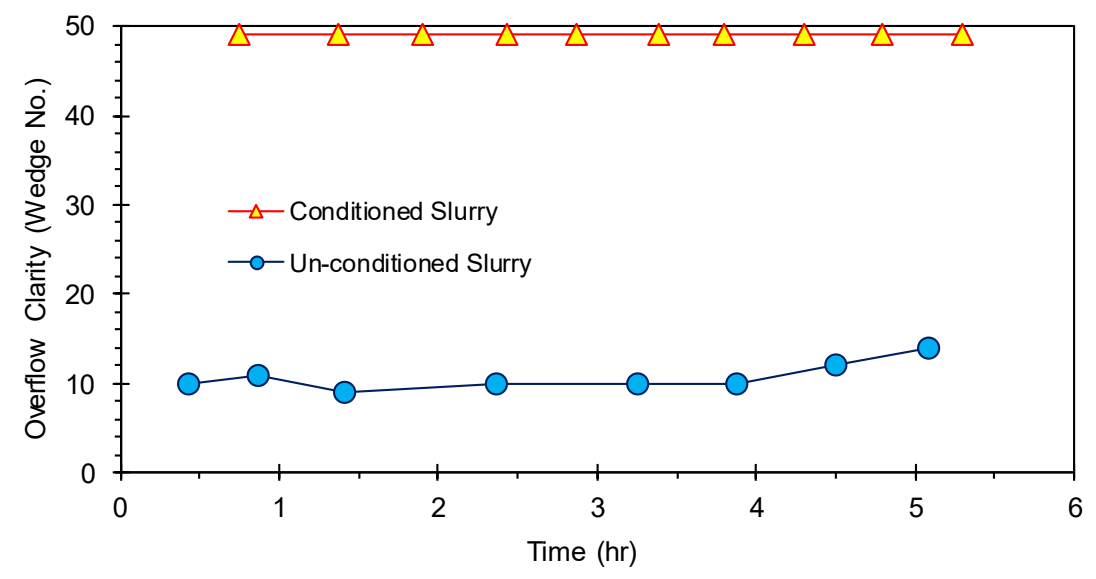

Figure 6 Comparison of overflow clarity between conditioned and unconditioned slurry

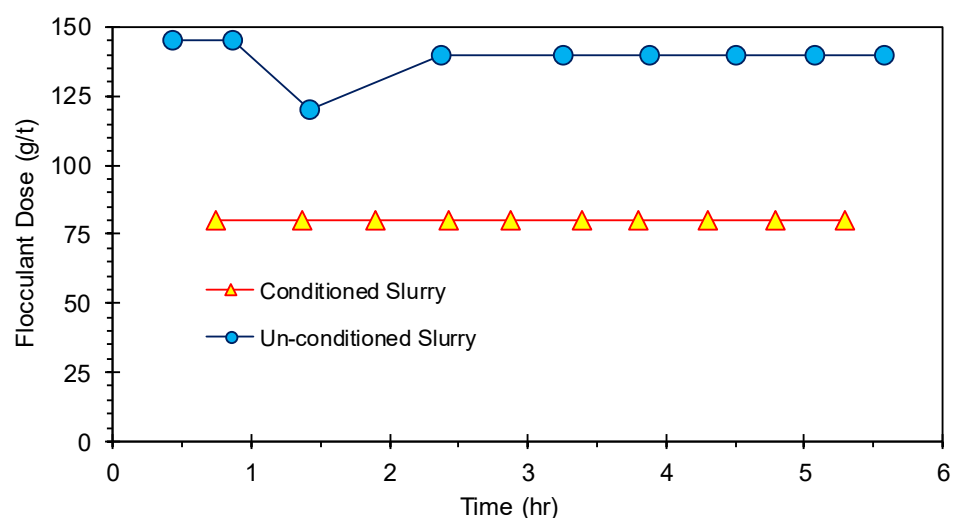

Figure 7 Comparison of flocculant dose between conditioned and unconditioned slurry

Conditioning of the process water did not appear to affect the underflow solids concentration achieved (Figure 8), however, it did influence the rheological properties of the underflow. The sheared yield stress of the conditioned slurry was higher than the unconditioned slurry (Figure 9), while the opposite appeared to be true for the viscous properties (Figure 10). 
Clay particles under coagulating conditions typically associate in edge-to-face orientations as opposed to face-to-face or edge-to-edge orientations which occur under dispersive conditions. The coagulated orientation provides greater inherent network strength to the slurry at rest and therefore, it is expected that the conditioned slurry would have a higher yield stress. Conversely, under shear, the conditioned particle packing may allow the slurry to obtain a lower viscosity than under the dispersive state.

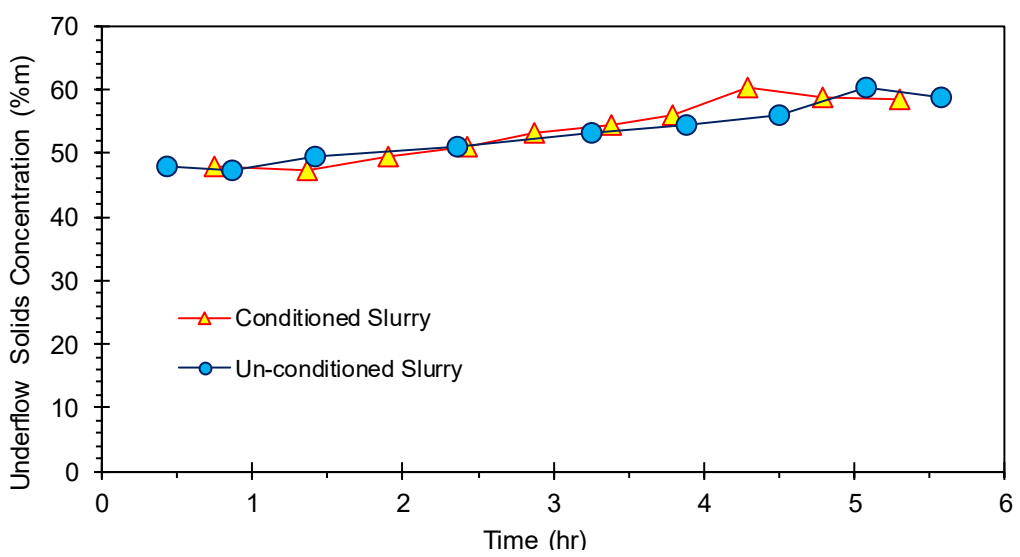

Figure 8 Comparison of underflow solids concentration between conditioned and unconditioned slurry

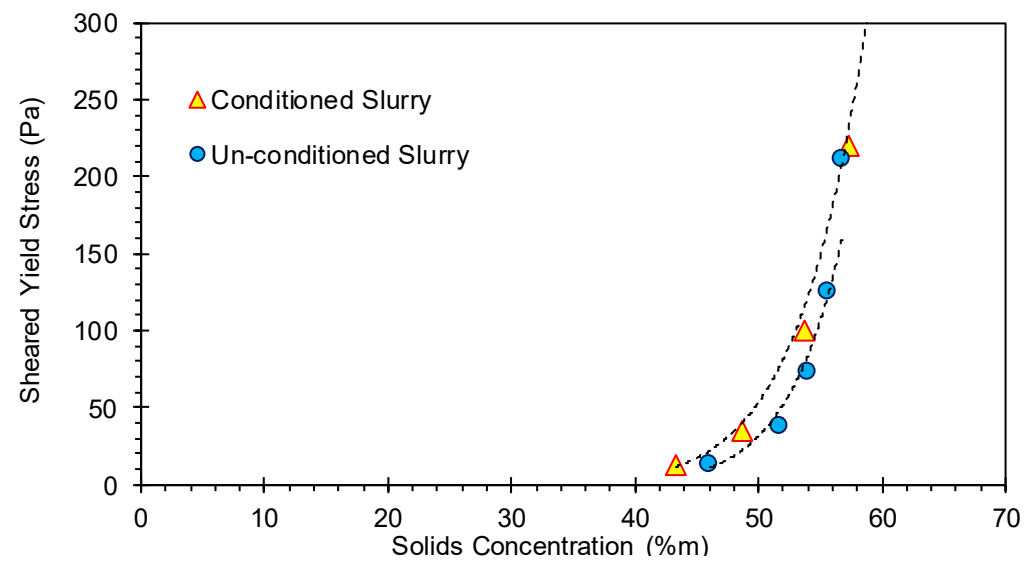

Figure 9 Comparison of underflow yield stress between conditioned and unconditioned slurry

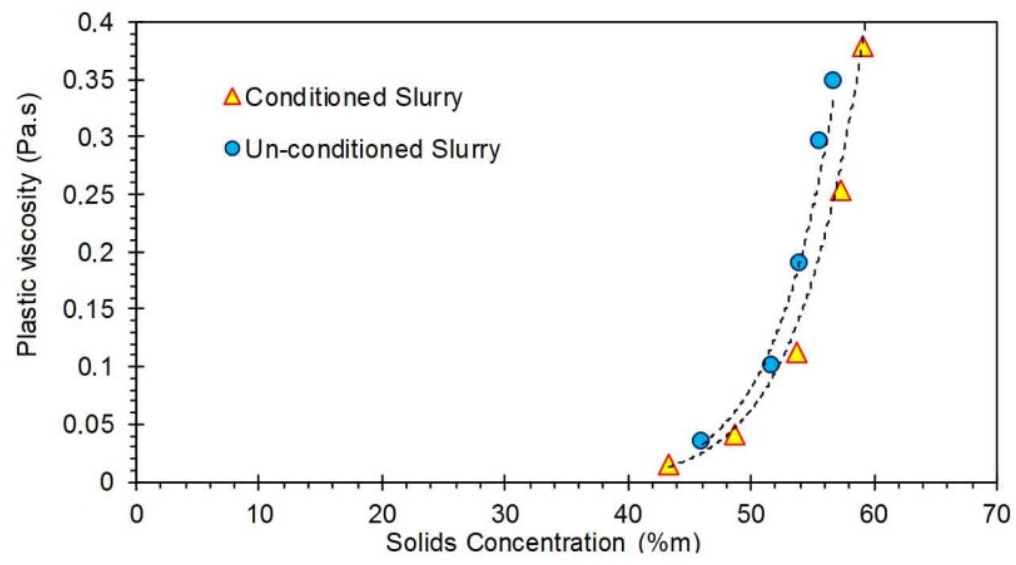

Figure 10 Comparison of underflow viscosity between conditioned and unconditioned slurry 


\subsubsection{Pressure filtration performance}

Within a diamond metallurgical circuit, it would be favourable to combine and dispose of both the thickened fines fraction (typically $-300 \mu \mathrm{m}$ ) with the barren grits fraction $(+300-1,000 \mu \mathrm{m})$. In an idealised flow sheet, the fines would be thickened in a paste thickener and the grits dewatered over a dewatering screen before being combined and filtered. The final filter cake would then be disposed of via a conveyor to a dry stacking area.

Although filtered tailings remain the ultimate goal, kimberlitic deposits which generate uncontrolled dispersive slurries are economically unfeasible to filter due to the high pressures, large filtration areas and low throughputs required. A trial was therefore conducted to determine if filtration could be made more feasible when treating a conditioned thickened product as compared to an unconditioned product.

Using the paste thickened underflow products $(-300 \mu \mathrm{m})$ from the previous tests, two filter feed slurries were generated by combining the thickened underflow with damp grits $(+300-1,000 \mu \mathrm{m})$ in a $1: 1$ dry solids mass ratio. The effect of the grits content on filtering was determined by preparing a conditioned sample with no grits as a control (Table 4).

Table 4 Pressure filter feed properties

\begin{tabular}{llll}
\hline Parameter & $\begin{array}{l}\text { Unconditioned } \\
\text { filter feed }\end{array}$ & $\begin{array}{l}\text { Conditioned } \\
\text { filter feed }\end{array}$ & $\begin{array}{l}\text { Conditioned filter } \\
\text { feed (no grits) }\end{array}$ \\
\hline Thickener underflow $(\% \mathrm{~m})$ & 48.6 & 47.7 & 47.7 \\
Yield stress of underflow $(\mathrm{Pa})$ & 14.4 & 32.4 & 32.4 \\
Mass of grits/litre underflow $(\mathrm{g})$ & 300 & 294 & - \\
Combined feed $(\% \mathrm{~m})$ & 56.3 & 55.7 & 47.7 \\
Filtrate conductivity $(\mathrm{mS} / \mathrm{cm})$ & 1.8 & 2.5 & 2.5 \\
\hline
\end{tabular}

All slurry types were dewatered in a $0.0176 \mathrm{~m}^{2}$ plate and frame filter at a feed pressure of 7, 10 and 15 bar; and a form time of 20 to 30 minutes. A filter cake could not be formed from the unconditioned slurry at both 7 and 10 bar pressure and the results of the trial are described in Table 5 and Figure 11 for the 15 bar feed pressure only.

Table 5 Filter results at 15 bar pressure

\begin{tabular}{llll}
\hline Parameter & $\begin{array}{l}\text { Unconditioned } \\
\text { filter feed }\end{array}$ & $\begin{array}{l}\text { Conditioned } \\
\text { filter feed }\end{array}$ & $\begin{array}{l}\text { Conditioned filter } \\
\text { feed (no grits) }\end{array}$ \\
\hline Filter cake appearance & Sloppy & Dry cake & Wet cake \\
Cake moisture $(\%)$ & 29.9 & 19.5 & 26.3 \\
Cake thickness $(\mathrm{mm})$ & 6.1 & 18.4 & 17.3 \\
Form time $(\mathrm{min})$ & 20 & 18 & 30 \\
Mass per unit area $\left(\mathrm{kg} \cdot \mathrm{m}^{2}\right)$ & 14.1 & 18.4 & 17.3 \\
\hline
\end{tabular}




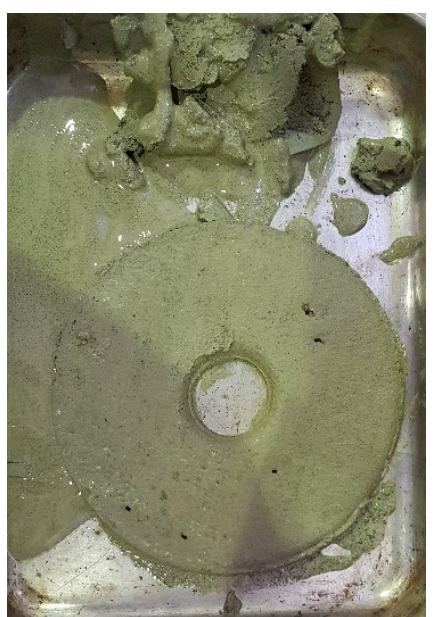

(a)

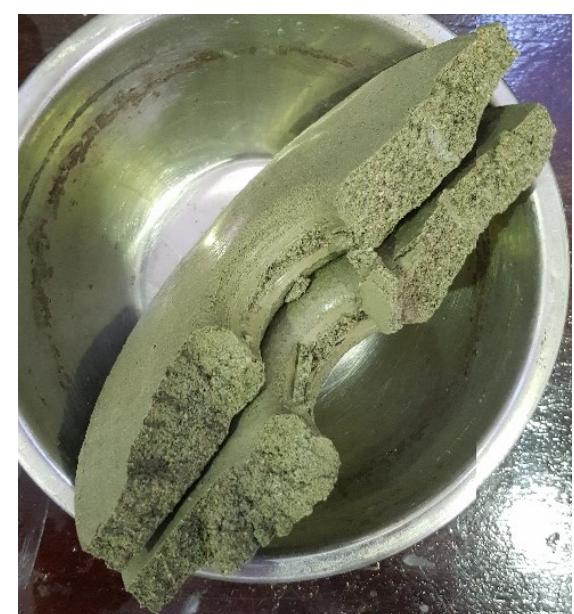

(b)

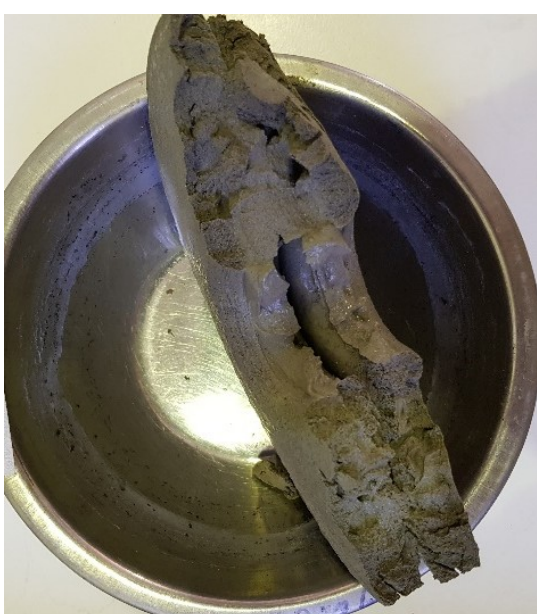

(c)

Figure 11 Appearance of filter cakes at 15 bar pressure. (a) Unconditioned feed; (b) Conditioned feed; (c) Conditioned feed without grits

The results showed that incorporating the coarse grits fraction into the thickened underflow had a positive effect on the filterability and dewatering ability of the slurry. However, the effect is significantly enhanced after conditioning of the thickener feed (Figure 12).

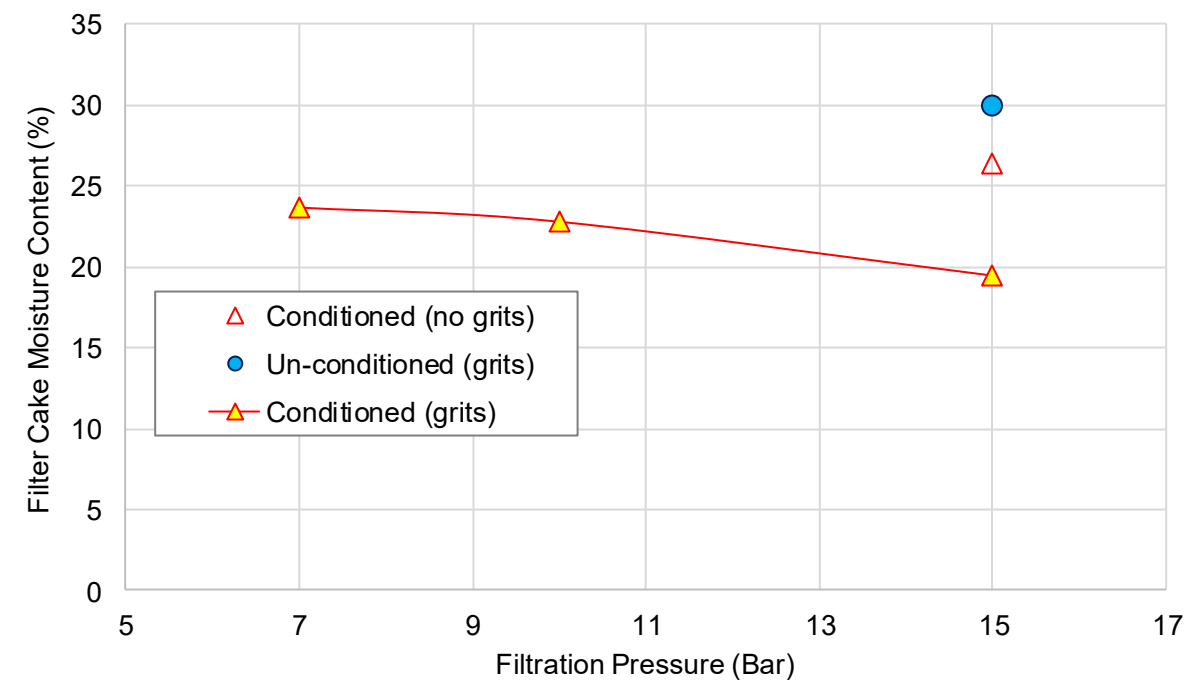

Figure 12 Relationship between feed pressure and filter cake moisture

The overall improvements in both paste thickening and pressure filtration dewatering performance by process water conditioning are quantified below in Table 6 . In terms of paste thickening, a $43 \%$ decrease in flocculant reagent operating cost along with clear overflow clarity was achieved. In addition, a $6.5 \%$ improvement in underflow solids concentration was realised.

Pressure filtration performance was also improved significantly with a 34.7\% reduction in filter cake moisture content and a $23.3 \%$ increase in tonnage throughput. However, these improvements were still not sufficient to make pressure filtration an economically feasible proposition for this specific tailings material. 
Table 6 Dewatering unit process performance improvements

\begin{tabular}{llll}
\hline Parameter & $\begin{array}{l}\text { Unconditioned } \\
\text { slurry }\end{array}$ & $\begin{array}{l}\text { Conditioned } \\
\text { slurry }\end{array}$ & $\begin{array}{l}\text { Performance } \\
\text { improvement }\end{array}$ \\
\hline Paste thickening & & & \\
Average overflow clarity (wedge no.) & 11 & 49 & Clear overflow \\
Average flocculant use (g/t) & 139 & 80 & $43 \%$ decrease \\
Average underflow density (\%m) & 50.2 & 53.7 & $6.5 \%$ increase \\
Pressure filtration (at 15 bar) & & & \\
Cake moisture (\%) & 29.9 & 19.5 & $34.7 \%$ decrease \\
Mass per unit area (kg. $\mathrm{m}^{2}$ ) & 14.1 & 18.4 & $23.3 \%$ increase \\
\hline
\end{tabular}

\section{Conclusion}

The occurrence of uncontrolled dispersive slurries in metallurgical plants can lead to poor efficiencies at the dewatering unit processes which will negatively impact the downstream tailings disposal and water recovery circuits.

Even more concerning is the fact that potentially viable dewatering options such as filtration will be dismissed and possibly excluded from flow sheet designs due to their poor performance, when tested against these types of slurries.

Process water conditioning to control the dispersive behaviour of clay containing slurries was shown to modify a potentially difficult slurry to one which was easily dewatered. The improvement in dewatering performance for both paste thickening and pressure filtration was quantified.

From a paste thickening perspective, conditioning the slurry showed a material decrease in flocculant operating costs, along with clear overflow water and a slight increase in underflow density and throughput. However, as would be expected from a coagulated slurry, the rheological properties were impacted with an increase in sheared yield stress and a decrease in plastic viscosity.

From a pressure filtration point of view, conditioning the slurry allowed it to be filtered to achieve a filter cake moisture of $19.5 \%$ as opposed to not being able to generate a dry cake at all. Although the filter throughputs are still uneconomic for this type of slurry, the principle of improved dewatering by filtration on a difficult clay slurry was shown.

\section{Acknowledgement}

The authors thank Debswana Diamond Company (Pty) Ltd for allowing this work to be published.

\section{References}

Mering, J 1946, 'The hydration of montmorillonite', Transactions of the Faraday Society, vol. 42, pp. 205-219.

Sequet, H, de la Calle, C \& Pezerat, H 1975, 'Swelling and structural organization of saponite', Clays and Clay Minerals, vol. 23, pp. 1-9.

Vietti, AJ 2018, 'A strategy for improving water recovery in kimberlitic diamond mines', Proceedings of the Diamonds - Source to Use 2018 Conference, The South African Institute of Mining and Metallurgy, Johannesburg. 